sensation, as if her heart was violently agitated, but so momentarily, that it goes as soon as it comes" (to use her own words), leaving her perfectly well. Perhaps the quantity of blood taken from a patient of so tender an age might surprise some of your numerous readers, yet, when we consider the certain relief afforded, and strength, not debility, produced by its abstraction, I think it was imperatively necessary in me to make free use of the lancet, with the view of relieving the irritability of the heart and the excited circulation; the digitalis seemed to act as a very efficient auxiliary.

\section{CASE OF CANCER OF THE BRAIN IN A CHILD.}

BY P. HENNIS GREEN, M.B.

CANCER of the brain is an extremely rare affection; it is still more rare in children than adults. The following is the only example which $I$ have seen in more than 800 cases of cerebral disease occurring amongst children :-

Philip Proteau, 12 years of age, was ad. mitted into hospital on the 18th May, 1833. For the last six months this child had experienced convulsive attacks confined to the right side of the face, trunk, and right arm; each access was preceded by headach and numbness of the arm, and recurred frequently; no other symptom of disease of the nervous system was perceived. The treatment first employed consisted in the ad. ministration of valerian, with cold and tepid baths. Soon after his admission, however, a number of boils appeared all over the body, and their existence seemed to exercise a salutary influence over the convulsive attacks. The latter diminished very considerably in number, but recurred on the disappearance of the boils, with their usual frequency. The patient was now sent to one of M. Guersent's wards, where the following symptoms were noted :-

After having experienced some headach for two or three hours, the boy felt some numbness in the right arm; lie then felt a sensation as if something ascended from the back of the hand to the chest, and imme. diately afterwards the limb became affected with convulsive movements. The muscles of the right eye, and of the right side of the face and chest, were also convulsed; the access lasted for two or three minutes and then ceased ; the patient never lost consciousness, nor presented any appearance of one labour. ing under epilepsy; during the convulsive movements he was able to answer with the utmost clearness any questions, but the articulation of sounds was slightly impeded.

The disease was judged to be a simple neurosis, and anti-spasmodics were liberally administered. The oxide of zinc, with extract of hyoscyamus, was first given, and the dose gradually augmented, until, on the 15th of July, the child took 30 grains of each dur. ing the day; cold baths were given at the same time. To these remedies the disease seemed to yield, and the accesses becoming much less frequent the child left the hospital on the 2nd of August; for the last 15 days he had been perfectly free from convulsive movements.

The cure, however, was only apparent; the convulsions returned in a few days after his departure, and he was readmitted on the 4th of September. He now remained in the hospital until the commencement of 1834. During this period the power of moving the right arm gradually diminished, and was, at length, completely lost; the boy frequently complained of pain in the left side of the head.

In this condition he continued until the 17th March, when he suddenly fell into a state of coma, accompanied by contracture of the limbs. He was immediately bled, but without any relief.

19. Face flushed; lips pale; complete coma; squinting of the right eye, the pupil of which is contracted, and motionless; left pupil dilated; upper extremities contracted; the forearm firmly flexed on the upper arm, this contracture being more strongly marked at the right side; the sensibility of that side, also, is diminished; skin cool ; pulse small, irregular, 76 ; respiration deep, regular, 20 ; tongue clean and moist; no vomiting or nausea; bowels constipated; abdomen retracted and free from pain; no trismus or other convulsive movement; purgative lavement; a seton in the neck. During the day, delirium, with plaintive cries.

On the 20th the symptoms continued without change.

On the 21st, skin cool; pulse regular, 144; respiration 20 ; right pupil extremely dilated, and insensible to the light; left pupil in its natural state; right arm firmly contracted; occasional delirium.

As the purgative lavement had no effect, two drops of croton oil were administered. These, also, producing no effect, two more drops were given, which brought away two copious evacuations during the night.

22. Pulse 132; right arm still contracted; in the night delirium, alternating with somnolence. Three drops of croton oil were administered.

23. The medicine has produced several stools; contracture of the right arm dimi. nished, but the loss of vision continues in the right eye; the patient now answers correctly when spoken to.

On the 24th and 25th this apparent im. provement continued, bnt on the 26 th he again became delirious; the face was highly 
fushed, and he cried constantly; two drops of croton oil were administered.

30. Complete coma; respiration stertorous; pulse insensible; sensibilty and motion entirely lost; strabismus. These nnfavourable symptoms followed the application of leeches behind the ears, and terminated in death at 7, P.M.

Body examined 38 Hours after Death.

Head.-Skull well formed. On the left side the dura mater adheres to the cerebral membranes, to the extent of two inches square. On the surface of the middle lobe of the left hemisphere, at the point where the dura mater adhered, is seen an encephaloid tumour, of the size of a hen's egg; it is irregular in form, inclosed in a cyst, and contains numerous bloody points not larger than pins' heads, but is not softened. The tumour extends into the lateral ventricle, and has destroyed the whole of the left corpus striatum. The nervous tissue surrounding the tumour, is completely diffluent, and behind there is a serous-looking cyst, which contains about three ounces of clear fluid. This cyst is merely separated from the ventricle by a layer of membrane resembling a serous one. The adjacent nervous tissue is but sligthly softened. Optic nerves intact; rest of the brain and spinal marrow healthy.

Chest.-The viscera contained in this cavity are quite healthy.

Abdomen.-The mucous lining of the great curvature of the stomach is softened. The mucous membrane of the small intestines is of a rosy colour, and somewhat softened also ; in several points they contain mucus, tinged with blood. The other viscera are healthy.

\section{THE HYDRENCEPHALOID AFFEC- TION OF INFANTS.}

\section{To the Editor of THE LANCET.}

Sir :-I should feel obliged by the insertion of the following remarks on the "hy. drencephaloid affection of infants," and its treatment, in your valuable Journal. Your obedient servant,

\section{5, Featherstone Buildings, Jan 30th, 1840.}

Edward Hocken.

Exhaustion, with coma, is much more liable to come on in infants after decided measures have been employed to stop the rapid and so frequently fatal progress of disorganization which arises from the inflammation of some important organ during the early periods of life, than is usually supposed. The inflammation, perhaps, is checked; the threatening symptoms of suffocation (if the lungs are affected) no longer remain; the congh has materially changed its character; the chest sounds well on percussion and auscultation, and, so far as the lungs are concerned, all the symptoms are proceeding favourably, and tending to a happy and speedy recovery.

But new, and, if possible, still more alarming symptoms, have made their ap. pearance on the subsidence of those of thoracic disease. The infant becomes affected with many signs, nearly resembling hydrocephalus; he lays pale, exhausted, and comatose, in the nurse's arms, but really sleepless, and be is readily roused; be frequently utters dismal and protracted groans, or a most unpleasant whining noise; the skin is cold, very pale, sometimes clam. my; the features collapsed, and very expressire of languor and anxiety; the pulse feeble, frequent, rarely intermittent, symp. toms which are much aggravated by any form of depletion, but ameliorated by nutri. tious food and stimulants. These indica. tions of exhaustion terminate rapidly ; if the proper treatment be withheld, or improper remedial measures employed, the depres. sion becomes greater, the pulse more rapid and feeble, and the child sinks into a fatally comatous condition. Dr. Gooch has, in two cases, however, seen, during the last few hours, symptoms of oppressed brain, such as coma, stertorous breathing, and dilated and motionless pupil.

This condition may come on from any cause of exhanstion besides loss of blood, as from diarrhoea, excessive purging, \&ci, as is the opinion of Dr. Marshall Hall and Dr. Abercrombie. Dr. Gooch, however, describes the affection as resulting without any apparent depleting or exhausting cause, and to be an idiopathic disease, the prominent symptoms of which are those of collapse and debility.

This hydrocephaloid affection so nearly simulates inflammation of the central parts of the brain, with effusion into the ventricles (bydrocephalus), that these two opposite pathological conditions have been, and in. deed are, frequently mistaken, and consequently improper treatment has been pursu. ed, to the great injury, if not to the certain death, of the unfortunate patient. How necessary is it, then, to discriminate them, to detect these deceptive symptoms, and trace them to their proper source; for, under the action of nutritious food, stimulants pro. portioned to the age and strength, and very minute and carefully regulated doses of laudd. num, the sinking infant revives, and once more gladdens its parents, to the credit of the physician's diagnosis and discernment, and his skill in remedial measures."

As regards the administration of minute doses of laudanum with nutritious food and stimulants, I may remark that it frequently proves of the most marked and decided

* Hocken on "Constitutional Influence in the Production of Disease." 